\title{
STRATEGY FOR THE CULTURAL CONTENTS INDUSTRY TO SECURE COMPETITIVE ADVANTAGE USING FOURTH INDUSTRIAL REVOLUTION TECHNOLOGY
}

\author{
Jin Sup Jung \\ Chungbuk National University \\ jsjung1@gmail.com
}

\section{Min Jae Lee \\ National Research Council for Economics, Humanities and Social Sciences fanmj85@naver.com}

\begin{abstract}
With the coming of the fourth industrial revolution, cultural contents industry requires a new strategy to enhance its competitive advantages. As a result, governments and companies have been focusing their efforts on finding new business models and expanding the value chain in order to respond to the paradigm shift in a timely manner. This study aims to suggest new business model frameworks and strategies to create a paradigm shift by converging the characteristics of the fourth industrial revolution and cultural contents industry such as publishing, game, broadcasting, and character industry. First, we discuss how to upgrade cultural contents industry with the fourth industrial revolution technology and then suggest new contents business models and the change of value chain in the cultural contents industry. Finally, we consider strategies of innovation process development in the cultural contents industry. This study will contribute not only to economic policy-making but also to the business strategy of the company.
\end{abstract}

\section{Keywords}

business model; competitive advantage; cultural contents industry; strategy; the fourth industrial revolution; value chain

\begin{abstract}
About the Authors
Jin Sup Jung received his Ph.D. from Seoul National University (South Korea). He is currently a Professor at the Department of International Business, Chungbuk National University (South Korea). His research focuses on foreign direct investment, corporate social responsibility, culture, and business model innovation. His research interests also include free trade agreement,
\end{abstract}


the 6th industrialization of agricultural, international culture, cluster, industrial and national competitiveness. He has published in such journals as Journal of Korea Trade (SSCI), Journal of Strategic Management, International Business Journal, International Business Review, and Korea Corporation Management Review. In addition, he serves as directors of many related academies such as Korean Academy of International Business, Korean Academy of International Business Management, Korean Society of Strategic Management.

Min Jae Lee is currently a Ph.D. candidate at the Department of International Business, Chungbuk National University. He serves as an Assistant Director at the National Research Council for Economics, Humanities and Social Sciences (NRC Korea). His research focuses on new business innovations and firm performances. His research interests also include business model innovations, MNC strategies, and creating shared values. His work has been published in journals such as Journal of Strategic Management, International Business Review, and Korea Corporation Management Review. In addition, his research was given the Best Paper Award of 2013 by the Maeil Business Newspaper, Korea Productivity Center. 
The wind of the fourth industrial revolution is hot. Beginning at the Davos Forum in 2016, this wind is breaking down the boundaries between industries based on advanced science and technology, creating new values, and emerging as an important issue of global economy (Schwab; Lee and Jung). Therefore, governments around the world are making efforts to establish a national strategy and find new growth engines to cope with the new era (UBS; WEF, The Future of Jobs; WEF, Digital Transformation). Especially, as the convergence of industries has been spreading rapidly, the linkages between industries and the development of related technologies are emerging as a key factor of national growth (Porter; Chesbrough, "Open Innovation"; Chesbrough, Open Innovation; Christensen; Rafols and Mayer).

The fourth industrial revolution means social, technological, and economic innovation that maximizes value creation by analyzing and utilizing accumulated online and offline data (Lee and Jung). This fourth industrial revolution not only connects and smartens devices and systems, but also extends to various fields from "gene sequencing" to nanotechnology, from renewable energy to quantum computing. As technology convergence-which is totally different to convergence of similar technology-has arisen among all industries, tremendous innovations and changes are taking place (Schwab). Accordingly, companies are making various efforts to develop and use science and technology that will lead the fourth industrial revolution such as autonomous vehicle, artificial intelligence, Internet of Things (IoT), energy source of next generations, biotechnology, and others. Governments are also launching a large-scale investment plan for the fourth industrial revolution-related technology and fields. In this regard, the cultural contents industry is attracting attention as the next generation growth engine. Not only is the cultural contents industry itself important as an independent industry, but it can also create value through linkage with other high-tech industries such as Information and Communications Technology (ICT) (Caves; Howkins; Banks and Hesmondhalgh).

Cultural content is a mixture of "culture" and "contents." It is a collective term in which contents with cultural elements are included in the media. In recent years, due to the advancement of fourth industrial revolution technology, new products and services, and customer values have emerged. Our society has also transformed into a society that emphasizes individuality. In the end, "soft power" rather than "hard power" ${ }^{2}$ has become an important factor for market and national competitiveness (Nye; Caves; Howkins; Park and Lee). In this respect, cultural contents are a product of human creativity, imagination, philosophy, sensibility, and reason that cannot be replaced by artificial intelligence or IoT, and when it is merged with other industries, the value of goods (or services) is developed to a higher and more important level. If so, what are the necessary competencies 
and competitive advantage strategies for sustainable growth and prosperity of the cultural content industry in the era of the fourth industrial revolution that we are facing now?

First of all, a clear understanding of the latest cultural contents industry and paradigm shifts in the business model of the fourth industrial revolution era should be foregrounded, and ultimately, we should have competence on cultural technology (CT). The fourth industrial revolution, that is just beginning, assumes that science technology and digitalization will completely change everything. This destructive change and innovation have been strongly felt because of the rapid progress of the fourth industrial revolution and the nature of its wide range, as well as the continuous convergence with many fields and the necessity of harmonization with the new environment resulting from it (Schwab). Therefore, in order to survive in the market and grow even more, companies and governments in the world should accurately recognize the meaning of these changes and actively deal with them.

This study focuses on the cultural contents industry, investigates the business model for securing competitive advantage, and suggests a new strategic paradigm in terms of enterprise and government. First, from the perspective of the enterprise, companies need to fundamentally review existing business models and make an "innovative business model" through detailed strategic direction and consistent practice. In particular, while the existing business model mainly focuses on how to effectively secure and deploy physical resources to generate revenue through cost savings, the business model of the fourth industrial revolution era, based on hyper-connectivity, hyper-intelligence, and hyper-convergence, will revolutionize existing business model and structures, and create competitive advantages by rebuilding an innovative process for upgrading added value (Lee and Jung).

On the other hand, the government should actively explore business models for the revitalization of the cultural contents industry, cultivate creative talents, and create related jobs. To this end, it is necessary to preemptively respond to the fourth industrial revolution through the establishment of mid- and longterm strategies and institutional improvement, and promote convergence among industries and technologies. Consequently, by linking the new contents created here with the fourth industrial revolution technology more closely, a virtuous cycle of high value-added ecosystem should be built, and it will contribute to the growth engine and new job creation of the new economy.

The structure of this paper is as follows. First, we will examine how the cultural contents industry should be developed in the fourth industrial revolution era. In particular, we will identify the key characteristics of the fourth industrial revolution, and examine how these characteristics are causing changes and innovations in the 
business model and value chain of the cultural contents industry. Next, we will discuss the necessary strategies for creating competitive advantages in the cultural contents industry, and propose strategies to create a virtuous cycle of "innovative business process." Finally, in the conclusion section, the significance, implications, and limitations of this study will be suggested.

\section{THE FOURTH INDUSTRIAL REVOLUTION AND THE CULTURE CONTENTS INDUSTRY}

\section{Key characteristics of the fourth industrial revolution}

Does the fourth industrial revolution really exist? And, if it exists, does it have a revolutionary distinction from the past? Some questions are still being discussed along with the advent of the fourth industrial revolution. The answers to these two questions are largely divided into three groups. First group has a negative theory about the fourth industrial revolution. They think that the innovation is not real (Gordon). They argue that there is no equivalent change in the name of the fourth industrial revolution, and that the latest development of digital technology is limited to certain areas and cannot be compared to technological innovations that happened in the second industrial revolution in the 1870s. This view is contrary to Schwab's argument that current changes are unprecedented tsunami levels in terms of speed, range, and impact. The second group agrees that "innovation really exists." However, they do not think that the fourth industrial revolution is clearly disconnected from the past. Therefore, they can be called the "reserves of the 4th industrial revolution" (Rifkin). The third group is the fourth industrial revolutionists, which thinks that the innovation is real and that the innovation has a disconnected character, making a new era (Schwab). The reason why such conflicting views appear in the same world can be interpreted in two ways. First, it is because the economics of the new technology have not yet been secured. As mentioned above, since the performance of new technology does not reach the performance of existing technology at present, the new technology does not reach the intersection exceeding the existing technology level, and the new technology has not spread fully.

Meanwhile, another point of view has recently been presented. This means that the front runner that quickly embodies new technology and achieves rapid technological innovation coexists with the laggards that are not able to innovate and apply the innovation to reality. It is also argued that the gap between the two is becoming wider. It is worth noting that both arguments are grounded in reality and that different perceptions of current changes directly affect the intensity and 
momentum of response. There is no consensus on the reality of the fourth industrial revolution, and its issues based on the discussions are as follows.

First, the most significant features that have emerged in the era of the fourth industrial revolution are "hyper-connectivity" and "hyper-intelligence," which are based on the dramatic increase in computational power and the vast amount of data validity. In addition, as these features obtain "convergence," business overcomes the constraints of time and space, and new values and changes emerge (WEF, The Future of Jobs). In other words, with the radical development and diffusion of ICT such as IoT and cloud computing, AI are exponentially expanding the connectivity between human beings, human beings and objects, objects and objects, and it becomes possible to grasp a certain pattern. It is also becoming possible to predict human behaviors based on these analysis results and create new businesses. The development of ICT and business model strongly combines the business of the virtual world with the business of the physical world, such as cyber physical systems (CPS). Therefore, the new business models using the characteristics of "hyper-connectivity," "hyper-intelligence," "hyper-convergence" have emerged (Lee and Jung). Therefore, first of all, this chapter examines the main characteristics of the fourth industrial revolution, the relationship among them, and the cultural contents industry (see Fig. 1).

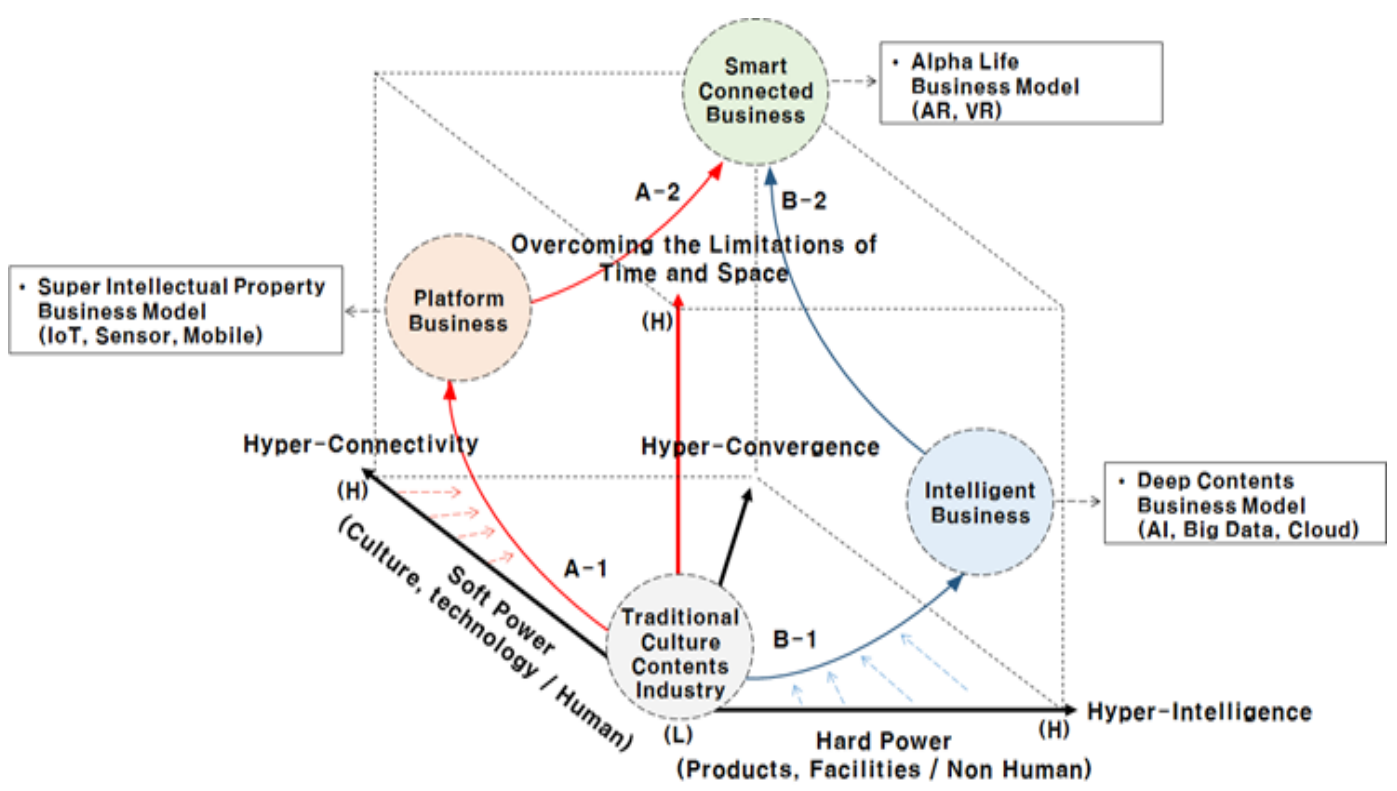

Fig. 1. Characteristics of the Fourth Industrial Revolution and Innovative Business Models in the Cultural Contents Industry 
Hyper-connectivity is the basic axis of future networks that bring all objects and objects to the network indefinitely by placing all heterogeneous networks, including people and objects, on a common platform. Thus, utilizing hyper-connectivity is at the core of the business model. That is, systematically, the objects and objects that make up the network are linked to the business and expanded through virtual platforms using digital technology.

Hyper-intelligence is the basic axis for optimizing a company's production structure and system such as quality improvement and productivity innovation by combining existing products and services with IoT, artificial intelligence, and sensors. Therefore, utilizing super-intelligence are at the core of the business model by combining big data and information with the cloud to analyze and think for themselves using AI or automation machine, thereby upgrading objects and objects in the digitized physical world.

Hyper-convergence is the basic axis for creating new values by recombining areas that have been separated due to hyper-connectivity and hyper-intelligence. For example, the convergence of digital and analog is Digilog, the fusion of cyber and reality is cyber physical system (CPS), and the fusion of product and service is product service system (PSS).After all, key to hyper-convergence is to optimize reality by recombining time and space in a virtual world where the real world is matched 1:1.

\subsection{A new business model for the cultural contents industry utilizing hyper-connectivity}

Hyper-connectivity means the disappearance and databaseization of the real and virtual boundaries according to the hyperlinked (or full online) based on the complete digitization (J. Kim; Yoo and Kim). In other words, hyper-connectivity means that the interconnections of people, organizations, and objects are increasing rapidly due to technological innovations such as the Internet, mobile technology, and the IoT, and all people and things are connected to networks by the development of digital technology (Quan-Haase and Wellman; Fredette et al.).

Since the cultural content industry is based on soft power, the more hyperconnectivity is enhanced, the faster the "platformazation" becomes, and the more efficient it becomes (J. Kim; Boudreau and Hagiu; Gawer and Cusumano). Then, when the related data is collected and accumulated, the linkage with hyperintelligence becomes easy. 
The platform was originally used to describe technologies that underlie the use of multiple products, either through interfaces with subsystems, or through the architecture of shared products (Robertson and Ulrich). However, as the development of ICT and related businesses have spread, it has become more and more popular to view the platform as a collection of access paths and interface solutions related to the problems faced by the subjects in the business ecosystem environment (Iansiti and Levien; Parker et al.). Therefore, platform business is a business that creates value through interaction between external producers and consumers. It provides an open participatory infrastructure that enables interactions among members through platform construction and finds optimal combination among users, which can be defined as a business that provides value to all the participants who make up the platform by facilitating the production and exchange of information (Chesbrough,; Cusumano and Gawer; Parker et al.).That is, the development of IoT and sensors will strengthen the human-object connection, which will lead to a great change in the production and consumption of cultural contents, beyond simple production and consumption, through the connection between all objects in society.

Like this, the fourth industrial revolution has spread the "experience economy," which enables cultural resources to be experienced regardless of whether the city or space is constrained by the provision of convergent media and contents (Pine and Gilmore). In particular, social interest and learning about "collective consumption" of media technology and contents are progressing at a rapid pace through the "fusion experience" in novelty of cultural contents (Kim and Kim). In addition, a variety of "intermediary platform of shared economic system" is formed through ICT, and a more complicated network system is structured online and offline. As a representative example, "Super intellectual property (IP)" is a combination of hyper-connectivity and the cultural contents industry.

The following is a description of the new business model in the cultural contents industry that utilizes hyper-connectivity. It is the "Super IP" business model that makes full utilization of the "platform business" through hyper-connectivity. Super IP is the creation of new and powerful IP assets by combining the elements of the fourth industrial revolution technology and business model into the elements of cultureandart, based on IP assets, which means "originality." This, in conjunction with the super platform that drives the digital distribution revolution, can dramatically increase business performance. For example, Korea's K-Pop is recognized as a Super IP, and it is possible to achieve excellent performance by managing a combination of content service, trade, commerce, and phantom community operation. The CJ E\&M company maximizes its performance by marketing through SNS accounts (Twitter, Facebook, etc.), sponsorship programs, MAMA awards ceremony, etc. in promoting "KCON event" to strengthen online-digital marketing. ${ }^{3}$ 
1.2 A new business model for the cultural contents industry utilizing hyper-intelligence

If the industrial revolution so far has changed the world by giving a huge amount of mechanical power to things, it is now moving into an era in which dramatic conversion is attempted by giving objects the ability to analyze and think for themselves. In this context, hyper-intelligence at the heart of the technology revolution and innovation is expected to solve problems that mankind has not solved and to greatly increase productivity.

Hyper-intelligence is a remarkable surpassing of people with superior ability in almost all areas of intelligence. This means that big data and information are combined with cloud, and the hardware itself analyzes and thinks itself so that the objects of the digitized physical world have intelligence that goes beyond the human being (Bostrom; Schwab). As a result, businesses are equipped with the help of state-of-the-art technology and ultimately become intelligent as the various functions are interconnected. In particular, these changes are rapidly spreading beyond automation, centering on the production sites of traditional businesses.

Namely, hyper-intelligence means "autonomization" of non-human agents due to the development of machine learning based on data acquired through hyperconnections, which strengthen the hard power of the cultural contents industry. In other words, analysis of data, development of artificial intelligence (AI) through deep learning, and expansion of machine-autonomy through it are the core of hyper-intelligence (Mitchell). Here, major changes are "personalization" and "machine-automation." As a result, a "personalized economy" is born, "replacement of human labor" occurs, and existing labor opportunities are reduced. Now is the time for "human-machine cooperation."

In the cultural contents industry, it is possible to show individualized movies, and artificial intelligence is increasingly producing contents directly. That is, AI directly creates various contents such as novels, movie scenarios, music, image contents trailers, and press articles. For example, newspapers such as The Los Angeles Times in the US are using artificial intelligence to write weather reports, sports news, and stock quotes. IBM's artificial intelligence, Watson, produced a trailer for the movie "Morgan" from 2oth Century Fox. In March 2017, "It's No Game" was announced, a short film by Benjamin, an AI writer. Such "Deep Content" is a representative example of the link between hyper-intelligence and the cultural contents industry.

The following is a description of the new business model in the cultural contents industry that utilizes hyper-intelligence. It is a "Deep Content" business model 
based on non-human hyper-intelligence using AI, big data, and the cloud. This is to reinforce knowledge information activities, such as deep learning and deep link, and to challenge the creation of new content that belongs to the domain of creation. This includes newspaper articles and digital textbooks written by robots. Google, Naver, and Cacao are increasing investment in the development of artificial intelligence technology and forecasting new content. For example, Naver, a Korean company, is gathering various AI technologies and is developing a combination of various modules and engines such as Natural Language Understanding (NLU), Neural Machine Translation (NMT), recommendation, and search engine data. Big data and AI will dramatically improve the search and utilization of content archiving, and accelerate production and commercialization of new contents in the domain of education and learning.

\subsection{A new business model for the cultural contents industry utilizing hyper-convergence}

Hyper-convergence is the creation of new values through convergence, fusion, or combination of domains that have been separated since the expansion of hyper-connectivity and hyper-intelligence. Examples of this are (i) onlineization of offline activities or convergence through them, (ii) convergence due to augmented reality and virtual reality, and (iii) convergence among other industries. In the cultural contents field, "the destruction of reality-virtual boundaries" is the most important conversion area. That is, the reality of the offline area and the online area meets in the virtual space or reality, and the boundary disappears in a way that the virtual world and physical reality recognizes each other through sight, hearing, and the like. The so-called virtual reality (VR) and augmented reality (AR) are core technologies of hyper-convergence. These "real-virtual and online-offline boundaries' disappearance and linkage and expansion" will dramatically upgrade the implementation and value of cultural contents.

The convergence of the industrial perspective in the cultural contents industry is largely divided into the convergence within industry and the convergence among industries. The convergence within the industry is a concept that is formed by the convergence of cultural technology $(\mathrm{CT})$ to the existing contents industry, and this content is called "digital contents." On the other hand, content that is created by combining CT with other industries as well as the existing content industry is called "convergent content." The Ministry of Culture, Sports and Tourism (MCST) in Korea regards the "convergence content" as a new type of digital contents industry that

can be easily connected with other industries such as manufacturing, service, and others through IT network based on IT technology. Convergent content is defined 
as futuristic content that includes knowledge, information, and various types of services beyond the existing entertainment content. Alpha Life, which is closely related to daily life, is closely related to this hyper-convergence.

The "Alpha Life" business model combines the above two segments and combines technologies such as AR, VR, and CPS to create a new content market tailored to the lifestyle of all humans. As mentioned above, deep content, using core technologies of AI and big data, and combined with AR and VR, etc., can be used in almost all areas such as general education and training, investment and insurance in daily life, healthcare, and personal protection. In other words, Alpha Life can support all lifestyle needs such as content usage, consumer life, learning, business activities, and leisure activities in the mobile ecosystem. For example, fashion content for a virtual experience fitting room will be commercialized at a large Mart clothing store. In addition, Online for Offline $\left(\mathrm{O}_{4} \mathrm{O}\right)$ uses this principle. It is a new concept business platform that combines the online database of an enterprise with offline activities, and the customer of the online company applies the database to the offline business. An example is "Amazon Go," which has an enormous database in the online distribution field, and makes inroads into the offline store business that can generate synergy.

\section{VALUE CHAIN INNOVATION IN THE CULTURAL CONTENTS INDUSTRY BASED ON THE FOURTH INDUSTRIAL REVOLUTION}

In general, the value chain refers to the entire range of activities from the conception of a product or service to the intermediate stage of production to distribution, use, and recycling to end consumers (Kaplinsky and Morris). Porter emphasized the importance of decomposing a company into strategically important activities, in order to understand the behaviors of cost leadership and differentiation, and the current or potential sources of competitive advantages. He also defined the value chain as total value-in other words, the sum of the profit (which is the effect) and activities (which is the cause) that forms these values. This perspective provides insights into the company's profit-making strategies. This is because it not only evaluates the competitiveness of enterprises in terms of added-value per activity, but also includes linkages with entities that directly or indirectly participate in the activities of the company (e.g., research institutes, universities, the government, and professional service providers).

The value chain analysis was developed to analyze the competitiveness of the industry (or a company) by dividing the economic activities created by the industry in stages, and it has several key characteristics. First, it provides the ability to 
analyze the profits generated through each activity by "mapping" various activities on the value chain. Second, it helps understanding the strengths and weaknesses of the industry not only through high value-added and low value-added activities, but also the mechanism of innovation creation through various activities in the value chain. In addition, it seeks to upgrade the relatively less competitive activities. Third, the unit of analysis can be extended to regional innovation systems (RIS) or national innovation systems (NIS), including "economic entities" (included within the value chain) as well as "institutions" that support them (Kaplinsky and Morris). Therefore, this value chain analysis can be widely applied to analyze the cultural contents industry of this study (Throsby), and the advantages of such analysis are as follows.

Value chain analysis focuses on all activities involved in delivering goods and services to customers from simple manufacturing and service (Wood; Pietrobelli and Rabellotti). As a result, it is possible to analyze the cultural contents industry, which has strong linkages between the manufacturing industry and service industry, as well as a vertical linkage in the production process and horizontal linkages, in a multifaceted and multi-layered value chain. In addition, the value chain analysis can dynamically look at the flow of added-value from production to distribution, unlike the existing quantitative industry analysis that analyzes the snapshot of the cultural production sector at a given point in time (Throsby). This makes it possible to analyze realistically the cultural contents industry that generates more added-value in the "intangible stage" such as distribution and marketing than the manufacturing stage (Park and Lee). In addition, value chain analysis facilitates the capture of the flow of knowledge as well as the flow of goods moving through the various stages of the value chain. Therefore, it is a suitable tool for analyzing the culture contents industry where knowledge, especially tacit knowledge, is more important than other industries. The value chain of the cultural contents industry can be divided into three stages: (i) creation and production, (ii) distribution and (iii) consumption (Pratt; Koivunen and Kotro; Hanzawa). Therefore, in the following, we will investigate how the technology of the fourth industrial revolution changes the various stages of the value chain in the cultural contents industry.

\section{i) The Creation and Production Stage}

The fourth industrial revolution is also revolutionizing the content creation stage in the value chain. The stage of creation corresponds to the research and development field in manufacturing industry as the basic stage of the cultural industry in which creators make creative cultural and artistic materials. In the past, the creation of contents has been the responsibility of creative writers and 
creators. However, at present, there are novels, paintings, and compositions created by artificial intelligence. Although the contents created by artificial intelligence still remain at the level of early imitation, AI is rapidly expanding its scope by producing new contents gradually and upgrading its level.

For example, in 2016, "Microsoft" and "Dutch researchers" published a project called "Next Rembrandt," where AI was taught deep-learning techniques of Rembrandt paintings and then painted. As a result, the work got a good reputation as the original. ${ }^{4}$ In Japan, a short novel written by AI passed the first prize in a literary award. In addition, a program has been developed to create music through a combination of vast amounts of music information.

In the tertiary industrial revolution, programming for automation was a major human role. However, in this way, in the fourth industrial revolution, how human beings utilize the ecosystem in which computers and machines communicate and create meaningful value is the main role of human beings. Therefore, in the era of the fourth industrial revolution, it is more important to integrate creative ideas with technology, knowledge, and products to create innovative businesses.

\section{ii) The Distribution Stage}

In the cultural contents industry, the distribution stage is the step of circulating the produced and manufactured cultural products to the consumers by means of media such as television, movies, and the Internet. In recent years, with the proliferation of the Internet and the progress of ICT technology, the cultural contents industry has been transformed into digitalized intangible contents, resulting in a new distribution structure. In particular, as the platform-based, hyper-connected content is distributed, and the curation service using the big data is activated, a customized content service is provided to consumers.

For example, Amazon launched the world's first audio recognition speaker "Echo" with the world's first artificial intelligence "Alexa" in 2014, and leading IT companies such as Google and Microsoft have also been competing in AI secretary speaker. In the case of Korea, SK Telecom's "Who (NUGU)" and KT's "GiGA Genie" are becoming more competitive. In addition, Netflix distinguishes 78, ooo consumer types based on big data, and uses AI to provide users with optimal movies. In Netflix, $75 \%$ of movie viewing is done through these referrals. 
Thus, in the era of the fourth industrial revolution, as all the information becomes more intelligent and the demands of the cultural users (consumers) are grasped in real time, it is necessary to understand their needs. In other words, technology for quickly grasping the needs of cultural users has become important as well as technologies for producing and providing contents at low cost.

\section{iii) The Consumption Stage}

Innovation from the fourth industrial revolution is changing the trends in the consumer sector and making consumers smarter. Looking at the changes in consumer trends, consumers are provided with tailored information by their needs, using technologies such as IoT and big data. Therefore, innovation in the consumer sector, which is supplied with products, is emerging automatically. For example, if the refrigerator is empty, the sensor automatically recognizes and communicates this to the consumer, and when the consumer chooses to buy the item, the smart refrigerator immediately places the order to the store.

In addition, the role of "prosumer" that builds contents using platform and customizes it to one's own taste is increasing. Therefore, contents in the era of the fourth industrial revolution are not being distributed by operators, but users are changing to "distribute" contents by processing, sharing, reconstructing, and mixing contents. Therefore, it is becoming more important to develop cultural contents that can integrate consumers' thinking, imagination, and ideas. Thus, the fourth industrial revolution brings a new paradigm of the cultural contents industry, culture, and consumer life. This new phase is beginning.

\section{STRATEGY FOR THE INNOVATION PROCESS DEVELOPMENT IN THE CULTURAL CONTENTS INDUSTRY}

In the fourth industrial revolution, business lies not only in physical reality but also in the virtual world. It also encompasses the development of new businesses by combining various industries (or fields and businesses), not just the simple manufacturing of products. Therefore, in order for traditional business to evolve into a business model in the era of the fourth industrial revolution, it is necessary to utilize science and technology to strengthen enterprise software (platform) and hardware (manufacturing process), and ultimately to realize a smart business

model. Ultimately, the "smart connected business model" will take advantage of 
advanced science and technology (ICT) to create an online and offline platform and increase the connectivity of people, things, and markets. In addition, it should be a circular structure that develops existing products, services, and business models by analyzing and processing the generated data and predicting and adapting them continuously (Lee and Jung).

In this section, we will examine the innovative business process of cultural contents industry using the "smart connected business framework." As we have seen, there are two ways to go to a smart connected business model. The first is to innovate the traditional business model by maximizing hyper-connectivity, and then to acquire hyper-intelligence and ultimately to merge the two characteristics. Another method is to maximize automation through hyper-intelligence of facilities, and then to secure hyper-connectivity using science and technology, and ultimately to converge the two characteristics. In summary, the approaches to reaching a smart connected business model are varied, but it is the convergence of the virtual world and real physical world by enhancing hyper-connectivity and hyper-intelligence. This will ultimately create a virtuous cycle of high value-added business models that will contribute to the growth of the new economy and new job creation.

Fig. 2 shows the results of the discussion. In order to innovate a business model by utilizing cultural contents, it is necessary to select industries to be integrated with cultural contents and use related technologies of the fourth industrial revolution. In addition, a virtual platform should be constructed to store, analyze, and utilize the collected data. The platform is complemented, enhanced, and distributed by connecting it to other platforms, enabling enterprises to optimize existing business models through analysis of the collected data. In the process of implementing such an optimized business model, the value chain is changed and new value is created. In particular, platforms can be connected and analyzed in the virtual world without time and space constraints, creating a whole new business from existing businesses. In other words, in the "smart connected business model," the business model is constantly innovated, and a new business is created through this virtuous cycle process.

For example, Netflix has developed algorithms that reflect consumers' personal preferences by closely analyzing and testing their subscribers' tastes, such as personalized content click histories, rental lists, and scores to provide consumercentric services. In addition, Netflix is producing content directly to provide exclusive content that is different from its competitors. In this way, when producing and planning contents, it is also analyzing the taste-related data of the viewers' consumption to produce contents that subscribers prefer. 


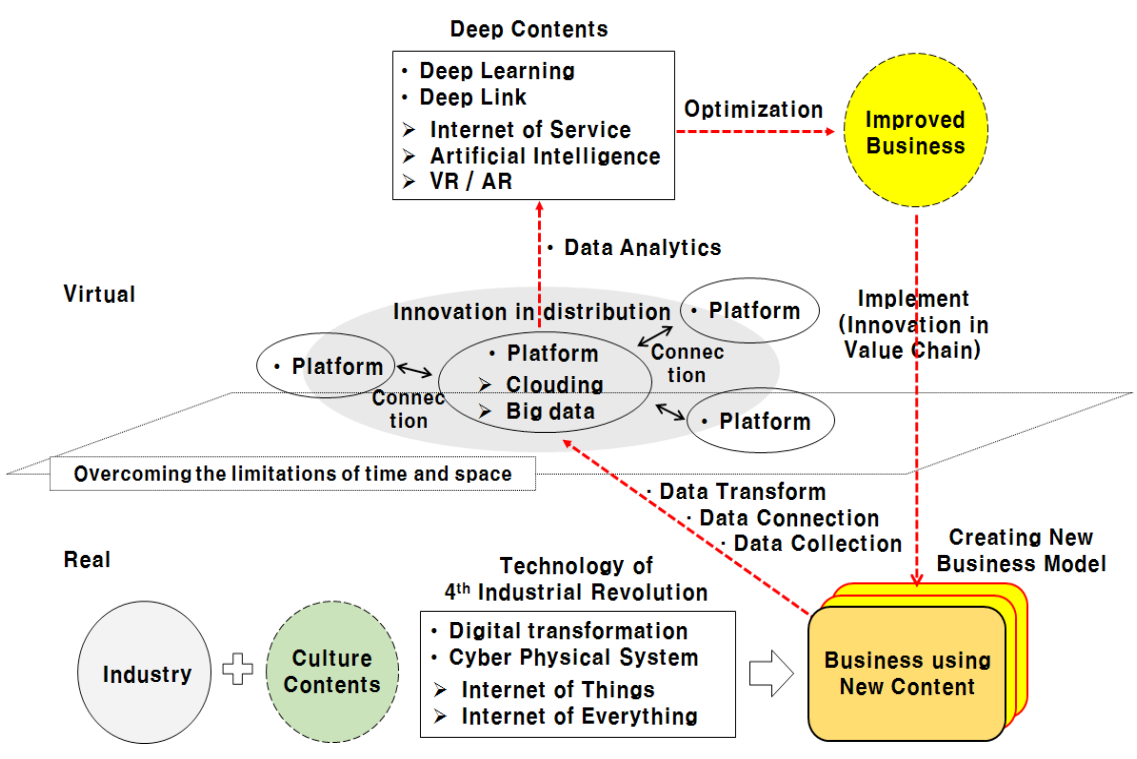

Fig. 2. Innovation of Business Process in the Cultural Contents Industry

\section{CONCLUSION}

Beyond time and space, humans and virtual animation coexist in movies, and museums can also be seen as VRs. ${ }^{5}$ In this way, the contents are combined with the technology of the fourth industrial revolution to enrich our lives and bring new interest, fun, and excitement. The cultural contents industry is at the center of the fourth industrial revolution. Therefore, recognizing these innovative technologies as cultural technology $(\mathrm{CT})$, we must "graft" the cultural contents industry to our lives and in existing industries, and have to nurture it. If Alvin Toffler, who foresaw an "information-oriented society" with a third wave, predicts the fifth wave, it is the society that drives human emotions. Therefore, emotional marketing is very important, and the core industry that changes human emotions is the cultural contents industry.

This paper is a study of technological types using advanced literature and deductive logic. It is an early study that applied the technology of the fourth industrial revolution to the cultural contents industry and upgraded the business model of the existing company to a more developed level. Thus, although there are various limitations, we can find the following strategic implications.

First, in this study, we defined the core of the fourth industrial revolution technology as hyper-connectivity, hyper-intelligence, hyper-convergence, and suggested some means to secure the competitive advantage of the cultural contents 
industry to meet these changes. In particular, we tried to explain the characteristics of the fourth industrial revolution technology and the latest new cultural contents business model based on it. The first is the "Super IP" business model that makes full use of the "platform business" due to hyper-connectivity. Second is the "Deep Content" business model based on non-human intelligence using AI, big data, and the cloud. Finally, the third is the "Alpha Life" business model, in which the above two segments are combined, and more technologies such as AR and VR are added. Here, a new content market (such as an individual customized market) can be created to suit all lifestyles in everyday life.

Second, this study examined what kinds of new changes are taking place in terms of the value chain of the cultural contents industry. As the technology of the fourth industrial revolution spreads throughout the cultural contents industry, the whole process of production and consumption is interconnected, and more intelligent and innovative changes are coming in which production and consumption interact closely with each other. In other words, until now, it has been a structure of the value chain in which a creative producer delivers value to consumers, but in recent years, value can be produced by artificial intelligence or consumers. Consumers and producers can also be interconnected, and the products distributed through a hyper-connected platform. A virtuous cycle system that can produce and consume at the same time is beginning.

Thus, the digital transformation of the contents industry has resulted in strengthening the influence of the platform. As a result, the globalization of the contents industry and the influence of the global platform are increasing, and an environment is being created in which users can easily find various contents made in other countries. However, because of the characteristics of contents, there are barriers due to the differences in cultural characteristics and preferences of various countries. Therefore, overcoming these is an important factor for the success or failure of the cultural contents industry.

Generally, there are two ways to go beyond cultural barriers. One is to minimize contents with cultural attributes in the content itself. This can be a strategy to prevent resistance to the content in advance, and it is typically a fantasy genre. The other is to actively reflect the cultural characteristics of the country in question on the contrary, or to create content in that country and go to new foreign markets. In addition to language, character design, user interface (UI), story, and other details to reflect the cultural characteristics of the "localization," the content can be enhanced through collaboration with local producers. Both have advantages and disadvantages, but in the former case, there are restrictions on the content genre and form, and others. Thus, if cultural attributes cannot be eliminated, the latter is preferred. 
Third, "innovation of business process in the cultural contents industry" is described in detail. In order for traditional business to evolve into a business model in the era of the fourth industrial revolution, it is necessary to utilize advanced science and technology to strengthen enterprise software (platform) and hardware (manufacturing process), and then to develop a predictable and customized virtuous system that evolves consistently.

On the one hand, as the use of artificial intelligence increases in all areas of content distribution, production, and consumption, issues surrounding these fields are also becoming important issues. This can be a legal and social controversy in situations where a clear definition or category of content or data involving artificial intelligence is not established. In other words, a large amount of data is required for artificial intelligence to function properly, and user data is included in the data security problem. Concerns about data leaks have also become an important issue as the data that algorithms need to analyze are becoming increasingly fragmented and increasing in quantity to provide personalized content.

On the other hand, the rapid technological change that accompanies the fourth industrial revolution is increasingly dismissing the traditional value chain of production of goods and services and increasing negative effects such as mass unemployment due to job losses (Ford; WEF, The Future of Jobs; WEF, Digital Transformation; Susskind and Susskind). Ford warned of the threat of a jobless future from the fourth industrial revolution, arguing that most jobs would be subdivided and replaced by a series of regular tasks. The WEF estimates that a total of 5.1 million jobs will disappear during the 2015-2020 period. Susskind and Susskind also warn that work done by professionals as well as manual workers can be replaced by machines. In this way, some people think that the fourth industrial revolution is considered to be more confusing than prosperity because human beings no longer have the opportunity to work in areas where robots and AI can do.

If so, is it possible to compromise between the technological realm and the human realm created by the fourth industrial revolution? It is clear that the changes caused by the fourth industrial revolution will bring efficiency and performance comparable to those of the existing industrial system. Until now, no one can predict the limitations of AI and big data related technology. In order to solve the problem, it is necessary to approach the new concept rather than the existing one.

The limitations of this study, which could be used as a follow-up study in future studies, are as follows. First, because this study is a deductive study, there are limitations on the methodology to some extent, and it can be supplemented by future empirical studies. Second, if there are more cases in the search for the business model of the fourth industrial revolution era, a framework with different 
characteristics may be found. In addition, strategies for detailed cultural contents industries such as games, characters, movies, publications, and broadcasting can be derived. Third, if the framework of this model is introduced and analyzed in other industries, different and meaningful implications can be derived. 


\section{Notes}

1. Professor Joseph Nye of Harvard University defined "soft power" as "cultural power including human reason and emotional ability such as education, culture, science, and technology" (256).

2. Generally speaking, "hard power" refers to the force expressed physically such as military or economic power. In this study, it refers to the advanced capability of tangible resources such as machine and manufacturing process.

3. In this case, KCON can be used as a Super IP.

4. Some people have a critical view of this project. The project, however, showed that data was used to make life more beautiful and the possibility in the creation of works that touch human souls.

5. See, for example, VR Museum of China (https://www.hiavr.com/news/ industry/39115.html). 


\section{Works Cited}

Banks, Mark, and David Hesmondhalgh. "Looking for Work in Creative Industries Policy." International Journal of Cultural Policy, vol. 15, no. 4, 2009, pp. 415-430.

Bostrom, Nick. Superintelligence: Paths, Dangers, Strategies. Oxford UP, 2014.

Boudreau, Kevin J., and Andrei Hagiu. Platform Rules: Multi-sided Platforms as Regulators. Edward Elgar Publishing, 2009.

Caves, Richard E. Creative Industries: Contracts between Art and Commerce. Harvard UP, 2000.

Chesbrough, Henry. "Open Innovation: A New Paradigm for Understanding Industrial Innovation." Open Innovation: Researching a New Paradigm, edited by H. Chesbrough, W. Vanhaverbeke, and J. West, Oxford UP, 2006, pp. 1-12.

--. Open Innovation: The New Imperative for Creating and Profiting from Technology. Harvard Business School Press, 2003.

Christensen, Clayton M. The Innovator's Dilemma: When New Technologies Cause Great Firms to Fail. Harvard Business School Press, 1997.

--. "The Ongoing Process of Building a Theory of Disruption." The Journal of Product Innovation Management, vol. 23, no. 1, 2006, pp. 39-55.

Cusumano, Michael, and Annabelle Gawer. "The Elements of Platform Leadership." MIT Sloan Management Review, vol. 43, no. 3, 2002, pp. 51-58.

Gawer, Annabelle, and Michael Cusumano. "Industry Platforms and Ecosystem Innovation." Journal of Product Innovation Management, vol. 31, no. 3, 2013, pp. $417-433$.

Ford, Martin. Rise of the Robots: Technology and the Threat of a Jobless Future. Basic Books, 2016.

Fredette, John, Revital Marom, Kurt Steinrt, Louis Witters, and Alcatel Lucent, “The Promise and Peril of Hyperconnectivity for Organizations and Societies," The Global Information Technology Report 2012, World Economic Forum, 2012.

Gordon, Robert. The Rise and Fall of American Growth: The U.S. Standard of Living since the Civil War. Princeton UP, 2017.

Hanzawa, Seiji. "Characteristics of the Division of Labor and Geography in Japanese Home Video Game Industry." Geographical Review of Japan, vol. 78, no. 10, 2005, pp. 607-633.

Howkins, John. The Creative Economy: How People Make Money from Ideas. Penguin, 2002.

Iansiti, Marco, and Roy Levien. The Keystone Advantage: What the New Dynamics of Business Ecosystems Mean for Strategy, Innovation, and Sustainability. Harvard Business School Press, 2004.

Kaplinsky, Raphael, and Mike Morris. A Handbook for Value Chain Research. Institute of Development Studies, University of Sussex and School of Development Studies, University of Natal, 2001.

Kim, Dae Ho. The Fourth Industrial Revolution. [in Korean]. Communication Books, 2016. 
Kim, Junic, "Platform Business and Network Strategy". STI Policy Review, vol. 5, no. 1, 2014, pp. 57-74.

Kim, Ki Youn, and Hung Kyu Kim. "A Study on Consumer Preference Typologing toward Sharing Economy Service Models Based on Collaborative Consumption: A Strategic Approach to Marketing Communications." Journal of Human Subjectivity, vol. 27, 2013, pp. 23-40.

Koivunen, Hannele, and Tanja Kotro. "A Value Chain in the Cultural Sector." Association for Cultural Economics International Conference, Barcelona, June 1998, pp. 14-17.

Lee, Min Jae, and Jin Sup Jung. "Competitive Strategy for Paradigm Shift in the Era of the Fourth Industrial Revolution: Focusing on Business Model Innovation." Indian Journal of Public Health Research \& Development, vol. 9, no. 8, 2018, pp. 357-362.

Nye, Joseph. S. "Soft Power and American Foreign Policy." Political Science Quarterly, vol. 119, no. 2, 2004, pp. 255-270.

Mitchell, Tom. Machine Learning. McGraw-Hill, 1998.

Park, Kyung Sook, and Chul Woo Lee. "The Impact of Value Chain Reorganization on Regional Economy: The Case of Daegu Cultural Contents Industry." Journal of the Economic Geographical Society of Korea, vol. 13, no. 4, 2010, pp. 601-622.

Parker, Geoffrey G., Marshall W. Van Alstyne, and Sangeet Paul Choudary. Platform Revolution: How Networked Markets Are Transforming the Economy and How to Make Them Work for You. Norton, 2016.

Pietrobelli, Carlo, and Roberta Rabellotti. Upgrading in Cluster and Value Chains in Latin America: The Role of Policies. Inter-American Development Bank, 2004.

Pine II, B. Joseph, and James H. Gilmore. The Experience Economy. Harvard Business Review Press, 2011.

Porter, Michael E. The Competitive Advantage of Nations. Macmillan, 1990.

--. Locations, Clusters, and Company Strategy: The Oxford Handbook of Economic Geography. Oxford UP, 2000.

Pratt, Andy C. "The Cultural Industries Production System: A Case Study of Employment Change in Britain, 1984-91." Environment and Planning A, vol. 29, no. 11, 1997, pp. 1953-1974.

Quan-Haase, Anabel, and Barry Wellman. Hyperconnected Network: Computer-mediated Community in a High-tech Organization. The Firm as a Collaborative Community, edited by C. Heckscher and P. Adler, Oxford UP, 2006, pp. 281-333.

Rafols, Ismael, and Martin Meyer. "How Cross-disciplinary is Bionanotechnology? Explorations in the Specialty of Molecular Motors." Scientometrics, vol. 70, no. 3, 2007, pp.633-650.

Rifkin, Jeremy. The Zero Marginal Cost Society: The Internet of Things, the Collaborative Commons, and the Eclipse of Capitalism. Palgrave Macmillan, 2015.

Robertson, David, and Karl Ulrich. "Planning for Product Platforms." MIT Sloan Management Review, vol. 39, no. 4, 1998, pp. 19-31.

Schwab, Klaus. The Fourth Industrial Revolution. Penguin, 2016.

Susskind, Richard, and Daniel Susskind. The Future of the Professions: How Technology Will Transform the Work of Human Experts. Oxford UP, 2016. 
Throsby, David. "Modelling the Cultural Industries." International Journal of Cultural Policy, 2008, vol. 14, no. 3, pp. 217-232.

Union Bank Switzerland. Extreme Automation and Connectivity: The Global, Regional, and Investment Implications of the Fourth Industrial Revolution. Union Bank Switzerland, 2016.

Wood, Adrian. "Value Chains: An Economist's Perspective." IDS Bulletin, vol. 32, no. 3, 2001, pp.41-45.

World Economic Forum (WEF). The Future of Jobs: Employment, Skills and Workforce Strategy for the Fourth Industrial Revolution. World Economic Forum, 2016.

-.. Digital Transformation Initiative Aviation: Travel and Tourism Industry. World Economic Forum, 2017.

Yoo, Young Sung, and Hyeon Jung Kim. The Advent of the Hyper-connected Society and Our Future. [in Korean]. (Gyeonggi Development Institute). Paju, Hanul Academy, 2014. 